\title{
Iatrogenic Cushing's syndrome caused by intranasal steroid use
}

\author{
Fatma Dursun, Heves Kirmizibekmez \\ Umraniye Training and Research Hospital, Istanbul, Turkey
}

\begin{abstract}
Cushing's syndrome (CS) is common after oral steroid use and has also been reported following topical or inhaled use, but it is extremely uncommon after intranasal administration. This is the case of a 6-year-old child who developed Cushing's syndrome after intranasal application of dexamethasone sodium phosphate for a period of 6 months. Pediatricians and other clinical practitioners should be aware that high-dose and long-term nasal steroid administration may cause iatrogenic Cushing's syndrome characterized by complications of glucocorticoid excess as well as serious and even life-threatening complications of adrenal insufficiency.
\end{abstract}

Keywords: Children; iatrogenic Cushing's syndrome; nasal steroid.

$\mathrm{C}$ ushing's syndrome (CS) is defined as high glucocorticoid level due to any cause and is classified as adrenocorticotropic hormone (ACTH) dependent or independent. CS commonly occurs due to exogenous systemic glucocorticoid administrations for treatment [1]. CS following use of inhaled or nasal steroid is rare and has been primarily reported in AIDS patients on ritonavir (a potent inhibitor of hepatic cytochrome P450) who were simultaneously taking inhaled steroids such as fluticasone for reactive airway disease $[2,3]$. The authors seek to emphasize that patients and caregivers should be informed about posology, method, and duration of administration of steroids before treatment is initiated. This report is the case of a child who developed iatrogenic CS following intranasal use of dexamethasone.

\section{CASE REPORT}

A 6-year and 3-month-old boy was admitted to pediatric endocrinology because of excessive weight gain and hypertrichosis. He had been diagnosed with allergic rhinitis 6 months prior, and was receiving a mixture prepared with Onadron ${ }^{\circledR}$ ampule (dexamethasone sodium phosphate) and physiological serum by nasal route. The mixture had been prescribed in a dose of 3 drops, twice a day. This was about $0.4 \mathrm{mg} /$ day of dexamethasone and was administered regularly for six months. Physical examination revealed moon face, hypertrichosis on skin of face, arms, and upper back, and purple striae on the skin of thighs (Figure 1). Weight was 30 $\mathrm{kg}(+2 \mathrm{SDS})$, height was $120 \mathrm{~cm}(+0.6 \mathrm{SDS})$ and body mass index (BMI) was $20.8(+2$ SDS $)$. Blood 


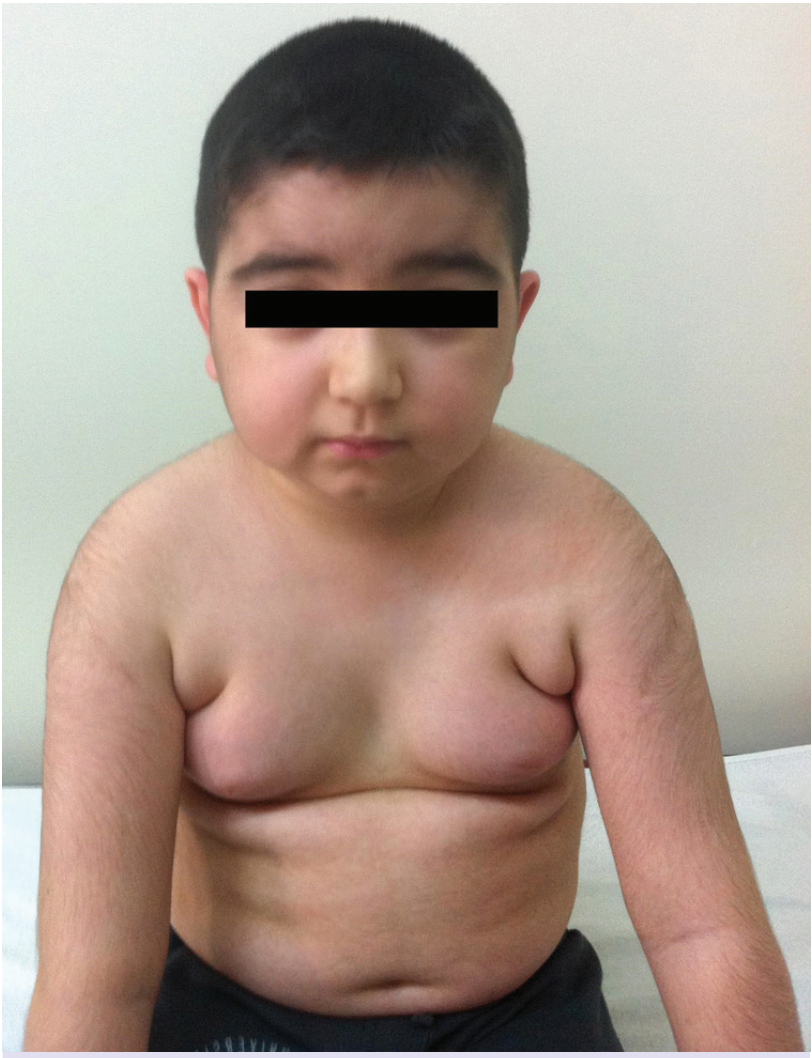

FIGURE 1. Physical appearance of the patient after using nasal steroid.

pressure and other findings of systemic inspection were normal. Early morning cortisol level was 2.1 $\mathrm{mcg} / \mathrm{dL}$, and serum transaminase levels were increased, while glucose, insulin, lipid profile and thyroid functions were normal. Low-dose ACTH stimulation test with $1 \mathrm{mcg}$ Synacthene was performed and stimulated cortisol level was found to be $3.4 \mathrm{mcg} / \mathrm{dL}$, suggesting hypothalamic-pituitaryadrenal (HPA) axis suppression.

Hydrocortisone was prescribed with a dose of 10 $\mathrm{mg} / \mathrm{m}^{2} /$ day for physiological replacement. Weight gain had ended, moon face was regressing and transaminase levels were in normal ranges 1 month after cessation of dexamethasone drops (Figure 2). Low-dose ACTH test was repeated at the end of 3 months, following a suspension of hydrocortisone replacement for 48 hours. HPA axis was still suppressed, so physiological hydrocortisone replacement was continued. The stimulated level of cortisol was normal at the end of 6 months.

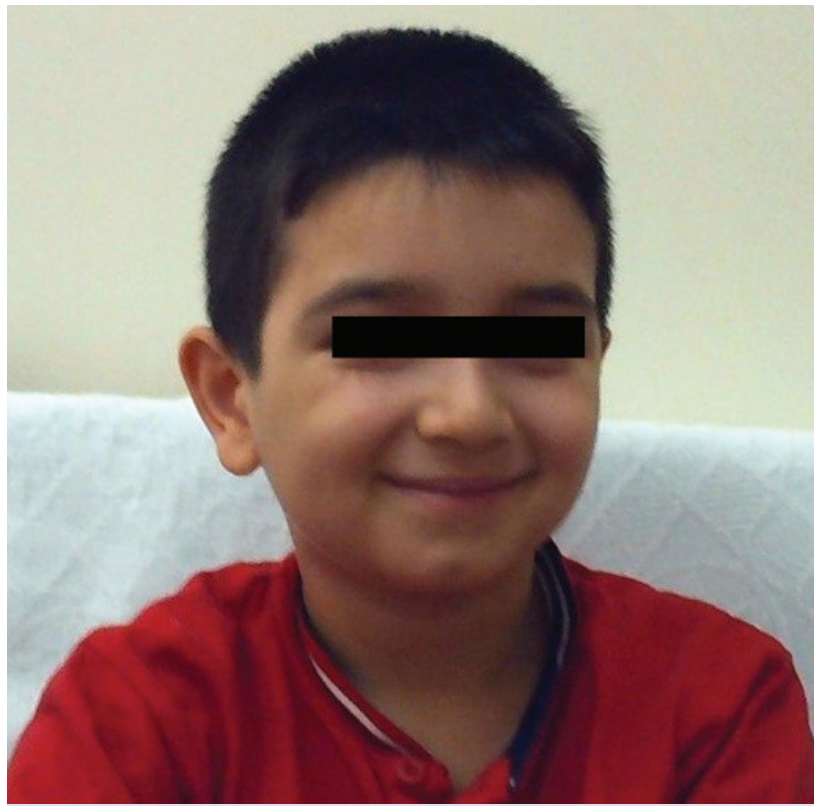

FIGURE 2. Moon face began to regress after the cessation of dexamethasone drops.

\section{DISCUSSION}

CS following use of intranasal steroids is rare [2, 4, 5]. Perry et al. [6] presented a series of 9 children with CS following use of steroid nasal drops (betamethasone, beclomethasone, fluticasone and flunisolide) for ear, nose and throat problems. Seven of the patients had received intranasal betamethasone, which was replaced with flunisolide in one. The other two patients were given budesonide and beclomethasone [6]. The present patient had received dexamethasone drops. CS following intranasal administration of dexamethasone for allergic rhinitis has also been reported previously [7].

Absorption through nasal mucosa and partly through intestinal mucosa after a portion of the dose is swallowed is the mechanism of systemic effect. Treatment consists of discontinuation of intranasal steroid preparation and tapering doses of hydrocortisone to account for secondary adrenal insufficiency until the axis recovers [7]. Normal daily cortisol production in the body ranges from 8 to 15 $\mathrm{mg} /$ day [8]. Wilkins and coworkers observed more than 40 years ago [9] that prednisone and dexamethasone are, respectively, 10 - and 80 -fold more 
potent than hydrocortisone in suppressing adrenal androgen production. The present patient had received $0.4 \mathrm{mg} /$ day dexamethasone, equal to approximately $32 \mathrm{mg} /$ day hydrocortisone. The stimulated level of cortisol became normal at the end of sixth month of the follow-up period.

When adrenal suppression from intranasal steroids is suspected, the most appropriate and widely available test is low-dose stimulation test with synthetic ACTH. HPA axis suppression was confirmed with low-dose ACTH test in the present patient. A non-invasive alternative is measurement of cortisol in salivary profiles, but currently, this assay is not routinely available [6]. If patients are treated with glucocorticoids at higher doses than the physiological dose for longer than 4 weeks, secondary adrenal cortex atrophy may develop as a result of inhibition of corticotropin-releasing hormone $(\mathrm{CRH})$ and $\mathrm{ACTH}$ secretions. Average 6 -week period is needed to restore the gland secretory function after discontinuation of the drug [1]. However, the current patient required physiological glucocorticoid replacement for 6 months to prevent adrenal insufficiency due to HPA suppression. In conclusion, pediatricians and other clinical practitioners should be aware that high-dose and long-term nasal steroid administration may cause iatrogenic CS. Iatrogenic CS is characterized by complications of glucocorticoid excess, as well as serious and even life-threatening complications of adrenal insufficiency.

Conflict of Interest: None declared.

Financial Disclosure: The authors declared that this study has received no financial support.

Authorship contributions: Concept, F.D.; Design, F.D.; Supervision, F.D., H.K.; Materials, F.D.; Data collection and/ or processing, F.D.; Analysis and/or interpretation, F.D., H.K.; Literature search, F.D., H.K.; Writing, F.D., H.K.; Critical review, F.D., H.K.

\section{REFERENCES}

1. Baş VN, Cetinkaya S, Aycan Z. Iatrogenic Cushing syndrome due to nasal steroid drops. Eur J Pediatr 2012;171:735-6.

2. Dutta D, Shivaprasad KS, Ghosh S, Mukhopadhyay S, Chowdhury S. Iatrogenic Cushing's syndrome following short-term intranasal steroid use. J Clin Res Pediatr Endocrinol 2012;4:1579.

3. Mahlab-Guri K, Asher I, Gradstein S, Zung A, Radian-Sade $\mathrm{S}$, Elbirt D, et al. Inhaled fluticasone causes iatrogenic cushing's syndrome in patients treated with Ritonavir. J Asthma 2011;48:860-3.

4. Fuchs M, Wetzig H, Kertscher F, Täschner R, Keller E. Iatrogenic Cushing syndrome and mutatio tarda caused by dexamethasone containing nose drops. [Article in German] HNO 1999;47:647-50. [Abstract]

5. Oluwayemi IO, Oduwole AO, Oyenusi E, Onyiriuka AN, Abdullahi M, Fakeye-Udeogu OB, et al. Iatrogenic Cushing's syndrome in children following nasal steroid. Pan Afr Med J 2014;17:237.

6. Perry RJ, Findlay CA, Donaldson MD. Cushing's syndrome, growth impairment, and occult adrenal suppression associated with intranasal steroids. Arch Dis Child 2002;87:45-8.

7. Kimmerle R, Rolla AR. Iatrogenic Cushing's syndrome due to dexamethasone nasal drops. Am J Med 1985;79:535-7.

8. Esteban NV, Loughlin T, Yergey AL, Zawadzki JK, Booth JD, Winterer JC, et al. Daily cortisol production rate in man determined by stable isotope dilution/mass spectrometry. J Clin Endocrinol Metab 1991;72:39-45.

9. Wilkins W, Thomas CC, Springfield, Ill. The diagnosis and treatment of Endocrine. Disorders in childhood 1950. 